\title{
Thermodynamic Bethe Ansatz for Biscalar Conformal Field Theories in Any Dimension
}

\author{
Benjamin Basso, ${ }^{1, *}$ Gwenaël Ferrando, ${ }^{1,2, \dagger}$ Vladimir Kazakov ${ }^{1,3,+}$ and De-liang Zhong $\oplus^{4, \S}$ \\ ${ }^{1}$ Laboratoire de physique de l'École normale supérieure, ENS, Université PSL, CNRS, Sorbonne Université, Université Paris-Diderot, \\ Sorbonne Paris Cité, 24 rue Lhomond, 75005 Paris, France \\ ${ }^{2}$ Institut de Physique Théorique, Université Paris-Saclay, CNRS, CEA, 91191 Gif-sur-Yvette, France \\ ${ }^{3}$ Theoretical Physics Department, CERN, 1211 Geneva 23, Switzerland \\ ${ }^{4}$ School of Physics and Astronomy, Tel Aviv University, Ramat Aviv 69978, Israel
}

(Received 2 April 2020; accepted 3 August 2020; published 24 August 2020)

\begin{abstract}
We present the TBA equations for the exact spectrum of multi-magnon local operators in the $D$-dimensional bi-scalar fishnet CFT. The mixing matrix of such operators is given in terms of fishnet planar graphs of multiwheel and multispiral type. These graphs probe the two key building blocks of the TBA approach, the magnon dispersion relation and scattering matrix, which we obtain by diagonalizing suitable graph-building operators. We also obtain the dual version of the TBA equations, which relates, in the continuum limit, $D$-dimensional graphs to two-dimensional sigma models in $\mathrm{AdS}_{D+1}$.
\end{abstract}

DOI: 10.1103/PhysRevLett.125.091601

Introduction.-The fishnet conformal field theory (FCFT) [1] (for a review see Ref. [2]) arises as a double scaling limit of weakly coupled and strongly $\gamma$-twisted $\mathcal{N}=4$ SYM theory. It stands out as a striking example of a nonsupersymmetric and yet integrable planar CFT in four dimensions, with an exactly marginal coupling $[3,4]$ and a nontrivial moduli space of vacua [5]. Because of these features, it has attracted a growing interest over the last few years [2,6-22]. Moreover, unlike its supersymmetric parent, the theory can be defined in any dimension $D$ $[23,24]$, called here $\mathrm{FCFT}_{D}$, with the Lagrangian

$$
\begin{aligned}
\mathcal{L}= & N_{c} \operatorname{Tr}\left[X^{\dagger}\left(-\partial_{\mu} \partial^{\mu}\right)^{\tilde{\delta}} X+Z^{\dagger}\left(-\partial_{\mu} \partial^{\mu}\right)^{\delta} Z\right. \\
& \left.+(4 \pi)^{\frac{D}{2}} \xi^{2} X^{\dagger} Z^{\dagger} X Z\right],
\end{aligned}
$$

where $X$ and $Z$ are two $N_{c} \times N_{c}$ matrix complex scalar fields with respective bare dimensions $\delta$ and $\tilde{\delta}=D / 2-\delta$ for $0<\delta<\frac{D}{2}$.

In the planar (large $N_{c}$ ) limit its perturbation expansion is dominated by conformal "fishnet" Feynman graphs: at high order the bulk structure of such graphs has the shape of regular square lattice with $X$ and $Z$ propagators pointing in two orthogonal directions [25]. First evidence for integrability came from Zamolodchikov, who treated the fishnet graphs with an appropriate choice of $D$-dimensional propagators as an integrable statistical mechanical system [24]. The integrability of the fishnet graphs is also closely

Published by the American Physical Society under the terms of the Creative Commons Attribution 4.0 International license. Further distribution of this work must maintain attribution to the author(s) and the published article's title, journal citation, and DOI. Funded by SCOAP . related to the integrability of conformal, noncompact $S O(1, D+1)$ spin chain with spins in principal series representations [26].

Quantum integrability of a QFT (defined, in a broad sense, as the existence of infinitely many conserved charges) usually allows for a deep insight into its nonperturbative structure. It also provides us with the tools for some explicit (though not necessarily easy) calculations of basic physical quantities, such as the correlators of local operators. A remarkable progress in this direction has been achieved in the last 15 years in the most emblematic planar integrable $\mathrm{CFT}-\mathcal{N}=4 \mathrm{SYM}$ [27-33]. In particular, the computation of the spectrum of anomalous dimensions of local operators (encoded in the two-point functions) appeared to be possible via the thermodynamic Bethe ansatz (TBA) [34-36], which finally evolved into the most efficient method of quantum spectral curve (QSC) $[37,38]$.

In this Letter, we propose TBA equations for $\mathrm{FCFT}_{D}$, at any $D$, for the dimensions of multi-magnon operators of the type

$$
\mathcal{O}_{J, M}(x)=\operatorname{Tr}\left(X^{M} Z^{J}\right)+\cdots
$$

The mixing matrix of such operators is entirely defined by multiwheel or multispiral planar Feynman graphs $[1,6]$, such as those on Fig. 1. These integrals have attracted considerable interest in the literature as examples of explicitly calculable multiloop Feynman graphs [10,15,39-41].

In the case of $\mathrm{FCFT}_{4}$, these TBA equations can be obtained by taking the double-scaling limit of the full TBA system of twisted $\mathcal{N}=4 \mathrm{SYM}[6,15,42]$. We no longer have this luxury once we deal with $\mathrm{FCFT}_{D}$ with $D \neq 4$, which does not have its SYM "parent" [43]. However, we 

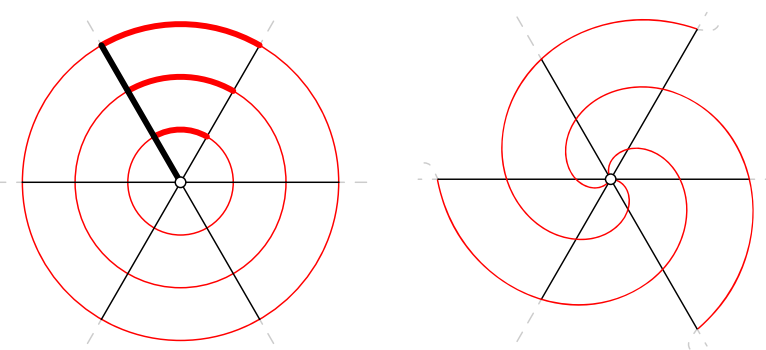

FIG. 1. Specimens of planar fishnet graphs contributing to the anomalous dimensions of multimagnon operators (central points) with black and red lines representing propagators of $Z$ and $X$ fields, respectively. Left panel: Multiwheel graph renormalizing the ground-state operator with $M=0$. The graph can be obtained by iterating the graph-building operator $\hat{\Gamma}_{N=3}$ shown here in bold face. Right panel: Multispiral graph contributing to the mixing of excited-state operators with $M=3$ magnons inserted at the origin.

can follow a different route and, as shown in the Letter, get the TBA equations more directly, by diagonalizing suitable graph-building operators. A generalization to excited states will allow us to derive asymptotic Bethe ansatz (ABA) equations for the dimensions of multimagnon operators, valid in the limit $J \rightarrow \infty$, and check them against explicit field theory computations. Lastly, following Ref. [44], we will explore our TBA at finite coupling, using a duality transformation, and find a correspondence between fishnet graphs and 2D nonlinear sigma models in $\mathrm{AdS}_{D+1}$ in the continuum limit, for any dimension $D$ and anisotropy $\delta$.

Graph-building operators and scattering data.-The TBA construction relies on the knowledge of the asymptotic data, dispersion relation and factorized $S$ matrix, that characterize the integrable structure of the fishnet graphs. In planar $\mathcal{N}=4$ SYM these were determined using supersymmetry and crossing symmetry [29,45-47]. We cannot follow these steps for FCFT ${ }_{D}$ for lack of symmetries but we can read off the scattering data from the graphs directly. In fact, the information can all be obtained from the wheel graphs shown in Fig. 1 (left) and corresponding to the local operator (2) with $M=0$, referred to as the vacuum state. General results for the excited states with $M \neq 0$ will be given in a subsequent section.

The $S$ matrix that is required here is the one controlling the scattering of magnons in the "open string channel" aka mirror kinematics. The idea is to treat the $X$ propagators along the angular direction in Fig. 1 as magnon excitations moving radially along the $Z$ propagators. Geometrically, this mirror one-dimensional system emerges from the decomposition $\mathbb{R}^{D} \cong \mathbb{R}_{+} \times S^{D-1}$, with $r=e^{\sigma} \in \mathbb{R}_{+}$being the distance to the origin, $\sigma$ the mirror position, and with the sphere $S^{D-1}$ giving rise to an internal $O(D)$ symmetry.
Mirror magnons evolve in this picture through the action of the graph-building operator

$$
\left[\hat{\Gamma}_{N} \Phi\right](\mathbf{x})=\int \Phi(\mathbf{y}) \prod_{i=1}^{N} \frac{\pi^{-D / 2} d^{D} y_{i}}{\left(x_{i-1}-x_{i}\right)^{2 \tilde{\delta}}\left(x_{i}-y_{i}\right)^{2 \delta}}
$$

with $x_{0}=0$ and $\Phi(\mathbf{x})=\Phi\left(x_{1}, \ldots, x_{N}\right)$. It acts on $N$-magnon wave function $\Phi$ in $\mathcal{H}_{D-\delta}^{\otimes N}$, where $\mathcal{H}_{D-\delta}$ is the representation space of the scalar representation of dimension $D-\delta$ of the conformal group in $D$ dimensions. This representation is unitary [48] and is part of the complementary series of the conformal group (see Ref. [49] for more details on these representations). Clearly, any wheel graph can be obtained by iteration of a graph-building operator; see Fig. 1. The significance of these operators in the fishnet theory was unveiled in Ref. [41] in the particular case $D=2$. Below we show how their diagonalization provides the $S$ matrix for the magnons for general $D$.

Magnon dispersion relation.- - Let us begin with the onemagnon problem, that is the diagonalization of the graphbuilding operator $\hat{\Gamma}_{N=1}$. This operator commutes with dilatation and rotations. As such, its eigenvectors have the form $x^{-2 \tilde{\beta}} C(x /|x|)$, where $C(y)=C^{\mu_{1} \ldots \mu_{l}} y_{\mu_{1}} \ldots y_{\mu_{l}}$ and $C$ is a symmetric traceless tensor of $\operatorname{rank} l \in \mathbb{N}$. A complete basis of states is obtained by taking $\tilde{\beta}=(D-\delta) / 2-i u$, with $u \in \mathbb{R}$, and choosing a complete basis of symmetric traceless tensors. One gets

$$
\int \frac{C\left(\frac{w}{|w|}\right)}{w^{2 \tilde{\beta}}(w-x)^{2 \delta}} \frac{d^{D} w}{\pi^{\frac{D}{2}}}=\lambda_{l}(u) \frac{C\left(\frac{x}{|x|}\right)}{x^{2(\tilde{\beta}-\tilde{\delta})}},
$$

where the eigenvalue is given by

$$
\lambda_{l}(u)=\frac{\Gamma(\tilde{\delta}) \Gamma\left(\frac{\delta}{2}+\frac{l}{2}+i u\right) \Gamma\left(\frac{\delta}{2}+\frac{l}{2}-i u\right)}{\Gamma(\delta) \Gamma\left(\frac{D-\delta}{2}+\frac{l}{2}+i u\right) \Gamma\left(\frac{D-\delta}{2}+\frac{l}{2}-i u\right)} .
$$

This eigenvalue is the weight of propagation of a magnon with rapidity $u$ and spin $l$, it naturally defines the magnon energy $\varepsilon_{l}$ through $\varepsilon_{l}=-\log \lambda_{l}$ while the momentum conjugate to $\sigma$ is $p_{l}(u)=2 u$ as can be read off directly from the expression of the eigenvector.

Magnon $S$ matrix.-For the magnon $S$ matrix, we proceed with the diagonalization of the graph-building operator of a two-frame wheel $\hat{\Gamma}_{N=2}$, which acts on functions of two variables. Global symmetries are no longer enough to solve the problem, but with only two magnons one can write the solution rather explicitly. We found that eigenvectors are given by

$$
\left\langle x_{1}, x_{2} \mid u_{1}, l_{1} ; u_{2}, l_{2} ; C\right\rangle=\int \frac{d^{D} x_{a}}{\pi^{\frac{D}{2}}} \frac{1}{x_{2 a}^{2\left(\tilde{\beta}_{1}-\frac{1}{2}\right)} x_{1 a}^{2\left(\tilde{\alpha}_{1}+\frac{l_{1}}{2}\right)}} C\left(\partial_{0}, \partial_{0^{\prime}}\right) \int \frac{d^{D} x_{b}}{\pi^{\frac{D}{2}}} \frac{x_{a b}^{-2\left(\alpha_{1}+\frac{D+l_{1}}{2}-1\right)}}{x_{0^{\prime} b}^{2\left(\tilde{\beta}_{2}-\frac{l_{2}}{2}\right)}} \frac{x_{0 b}^{2\left(\tilde{\beta}_{1}+\frac{l_{1}}{2}-1\right)}}{x_{01}^{2\left(\tilde{\beta}_{1}-\frac{l_{1}}{2}\right)}}
$$




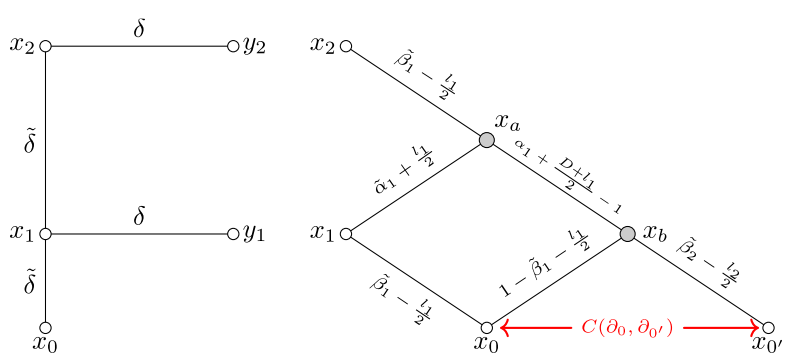

FIG. 2. Integral kernel of the graph-building operator $\hat{\Gamma}_{N=2}$ (left panel) and eigenvector (6) (right panel). The action of the former on the latter is explained in Eq. (3). Gray shaded points represent integration points while a line with index $\alpha$ connecting points $x$ and $y$ represents the propagator $(x-y)^{-2 \alpha}$.

evaluated at $x_{0}=x_{0^{\prime}}=0$ [50]; see Fig. 2 for a graphical representation of this function. Here $x_{i j}=x_{i}-x_{j}$, $\alpha_{j}=D / 2-\tilde{\alpha}_{j}=\delta / 2-i u_{j}, \quad \beta_{j}=D / 2-\tilde{\beta}_{j}=\delta / 2+i u_{j}$, and $C\left(y_{1}, y_{2}\right)=C^{\mu_{1} \ldots \mu_{l_{1}} \nu_{1} \ldots \nu_{l_{2}}} y_{1 \mu_{1}} \ldots y_{1 \mu_{1}} y_{2 \nu_{1}} \ldots y_{2 \nu_{l}}$, where $C$ is a tensor that is symmetric and traceless, separately, in the first $l_{1}$ indices and in the last $l_{2}$ ones. The eigenvalue associated with Eq. (6) can be computed directly using notably the star-triangle identity. It reads $\lambda_{l_{1}}\left(u_{1}\right) \lambda_{l_{2}}\left(u_{2}\right)$, which means that the total energy is the sum of the individual energies.

The full $S$ matrix can be read off from the asymptotics of these eigenvectors in the limit $x_{12}^{2} \rightarrow \infty$. In this limit, the mirror magnons are far apart from each other and their wave function reduces to the sum of incoming and outgoing plane waves

$$
\begin{aligned}
& \left(x_{1}^{2}\right)^{i u_{1}}\left(x_{2}^{2}\right)^{i u_{2}} C\left(\frac{x_{1}}{\left|x_{1}\right|}, \frac{x_{2}}{\left|x_{2}\right|}\right) \\
& +\left(x_{2}^{2}\right)^{i u_{1}}\left(x_{1}^{2}\right)^{i u_{2}}\left[\mathbb{S}_{l_{1}, l_{2}}\left(u_{1}, u_{2}\right) C\right]\left(\frac{x_{2}}{\left|x_{2}\right|}, \frac{x_{1}}{\left|x_{1}\right|}\right),
\end{aligned}
$$

up to an overall prefactor that depends on $x_{1}$ and $x_{2}$, and with, in square brackets, the tensor obtained by acting on $C$ with the $S$ matrix. We conjecture that the $S$ matrix obtained this way is given by

$$
\mathbb{S}_{l, l^{\prime}}(u, v)=\frac{f_{l}(u)}{f_{l^{\prime}}(v)} \mathcal{S}_{l, l^{\prime}}(u-v) \mathbb{R}_{l, l^{\prime}}(u-v),
$$

where the dynamical factors are

$$
\begin{aligned}
\mathcal{S}_{l, l^{\prime}}(u)= & \frac{\Gamma\left(1+\frac{l+l^{\prime}}{2}-i u\right)}{\Gamma\left(1+\frac{l+l^{\prime}}{2}+i u\right)} \frac{\Gamma\left(\frac{D}{2}+\frac{l+l^{\prime}}{2}+i u\right)}{\Gamma\left(\frac{D}{2}+\frac{l+l^{\prime}}{2}-i u\right)} \\
& \times \frac{\Gamma\left(\frac{\left|l-l^{\prime}\right|}{2}+i u\right)}{\Gamma\left(\frac{\left|l-l^{\prime}\right|}{2}-i u\right)} \frac{\Gamma\left(1+\frac{\left|l-l^{\prime}\right|}{2}+i u\right)}{\Gamma\left(1+\frac{\left|l-l^{\prime}\right|}{2}-i u\right)}
\end{aligned}
$$

and

$$
f_{l}(u)=\frac{\Gamma\left(\frac{\delta}{2}+\frac{l}{2}-i u\right) \Gamma\left(\frac{D-\delta}{2}+\frac{l}{2}-i u\right)}{\Gamma\left(\frac{\delta}{2}+\frac{l}{2}+i u\right) \Gamma\left(\frac{D-\delta}{2}+\frac{l}{2}+i u\right)},
$$

and with $\mathbb{R}_{l, l^{\prime}}$ the $O(D)$-symmetric $R$ matrix on the irreps of spins $l$ and $l^{\prime}$, with $\mathbb{R}_{l, 0}=1$. These $R$ matrices can be determined through their eigenvalues [51-53] or by fusing Zamolodchikov's $R$ matrix [54]

$$
\mathbb{R}_{1,1}(u)=\frac{u \mathbb{1}}{u+i}+\frac{i \mathbb{P}}{u+i}-\frac{i u \mathbb{K}}{(u+i)\left(u+i \frac{D-2}{2}\right)},
$$

with $\mathbb{1}, \mathbb{P}$, and $\mathbb{K}$ being identity, permutation, and contraction of two $D$-dimensional vectors.

We could partly verify our conjectures, Eqs. (7)-(10), by considering some particular cases for which we were able to compute the asymptotic behavior of the eigenvectors (using the method of expansion by regions [55]). This includes eigenvectors associated to completely symmetric traceless tensors $C$ of any rank $l_{1}+l_{2}$. They are eigenstates of $\mathbb{R}_{l_{1}, l_{2}}$ with eigenvalue 1 and gave us access to the functions $f_{l}$ and $\mathcal{S}_{l_{1}, l_{2}}$. We have also explicitly reproduced $\mathbb{R}_{1,1}$. This match gives confidence that the general conjecture is correct, as the latter $R$ matrix is the seed for the higher-spin ones. (Indeed, assuming integrability, Yang-Baxter relations entirely determine $\mathbb{R}_{l, l^{\prime}}$ given $\mathbb{R}_{1,1}$.) Nonetheless, it would be nice to check the matrix structure for higher spins from the general wave function (6).

For $D=2$, eigenvectors were found in Refs. [41,56] and we checked that their asymptotic behavior allows one to recover exactly the full $S$ matrix (8)-(10).

As a final check, for $D=4$ isotropic fishnets $(\tilde{\delta}=\delta)$, we verify agreement with the conjectured $S$ matrix of the $\mathcal{N}=4$ SYM theory [29] at weak coupling in the mirror kinematics. In fact, our analysis is the first field theory derivation of this mirror $S$ matrix.

TBA for ground state.-We turn to the scaling dimension $\Delta$ of operator $\mathcal{O}_{J, 0}$. It governs the divergent part of the wave function renormalization, $Z=\sum_{N \geq 0} \xi^{2 J N} \operatorname{tr}\left(\hat{\Gamma}_{N}\right)^{J}$, generating the wheel graphs, see Fig. 1, with the divergences arising from the trace over the magnons eigenstates. In the 1D picture, it corresponds to the free energy of a system of magnons at temperature $1 / J$ and chemical potential $\log \xi^{2}$. The factorization of the $S$ matrix permits its determination at any $J$ and $\xi$ using the TBA equations. Following wellknown saddle-point procedure [57-60], it takes the form

$$
\Delta=J \tilde{\delta}-\sum_{l \geq 0} \int p_{l}^{\prime}(u) \log \left[1+Y_{1, l}(u)\right] \frac{d u}{2 \pi},
$$

where $p_{l}^{\prime}(u)=2$ and where the $Y$ functions $Y_{1, l}$ describe the distribution of energy per magnon, with $l \in \mathbb{N}$ labeling the spherical harmonics.

The latter $Y$ functions are part of a larger family of functions $\left\{Y_{a, l>0}\right\}$ needed to account for the matrix degrees of freedom. For the sake of simplicity, we shall restrict 
ourselves to the simply laced case, corresponding to even dimensions $D>2$. Hence, $a \in[1, r]$ labels the nodes of the $O(D+2) \cong D_{r}$ Dynkin diagram, with $r=D / 2+1$ and incidence matrix $I_{a b}$.

The $Y$ functions themselves are determined by an infinite system of nonlinear TBA equations. Denoting $L_{a, l}=\log \left(1+Y_{a, l}\right)$, these equations take the form

$\log Y_{1, l}=C-J \varepsilon_{l}+\sum_{l^{\prime} \geq 0} \mathcal{K}_{l, l^{\prime}} \star L_{1, l^{\prime}}+\sum_{l^{\prime} \geq 1} K_{l, l^{\prime}} \star L_{2, l^{\prime}}$,

for the massive nodes $(a=1, l \geq 0)$, where $\varepsilon_{l}=-\log \lambda_{l}$,

$$
C=J \log \xi^{2}-\sum_{l=0}^{\infty} \int\left[i \partial_{u} \log f_{l}(u)\right] L_{1, l} \frac{d u}{2 \pi},
$$

and where the $\star$ operation denotes the convolution on the real axis (with measure $d u / 2 \pi$ ). For the remaining, auxiliary, nodes for spin excitations $(a>1, l \geq 1)$ the equations are

$$
\log Y_{a, l}=-\sum_{l^{\prime} \geq 1} \check{K}_{l, l^{\prime}} \star L_{a, l^{\prime}}+\sum_{b, I_{a b} \neq 0} \sum_{l^{\prime} \geq 1} K_{l, l^{\prime}} \star L_{b, l^{\prime}},
$$

where we introduced $\check{K}_{l, l^{\prime}}=K_{l, l^{\prime}+1}+K_{l, l^{\prime}-1}$, with symmetric kernels $\mathcal{K}, K$ defined by

$$
\mathcal{K}_{l, l^{\prime}}(u)=-i \partial_{u} \log \mathcal{S}_{l, l^{\prime}}(u),
$$

and

$$
K_{l, l^{\prime}}(u)=\sum_{j=\left(\left|l-l^{\prime}\right|+1\right) / 2}^{\left(l+l^{\prime}-1\right) / 2} \frac{2 j}{u^{2}+j^{2}} .
$$

Finally, let us stress that the kernels obey the universal asymptotics $\mathcal{K}_{l, l^{\prime}}(u)=2 \log u^{2}+O\left(1 / u^{2}\right)$ at large rapidity. Consequently, the scaling dimension (12) controls the asymptotics of the main $Y$ functions,

$$
\log Y_{1, l} \sim-\Delta \log u^{2} .
$$

The auxiliary $Y$ functions are, on the other hand, asymptotically constant at $u \rightarrow \infty$.

Dual TBA and Y system.-The TBA equations above give us a good handle on the scaling dimension at weak coupling, which is when the massive $Y$ functions are small and the equations are solvable iteratively. They are also very useful for the study of fishnet graphs at large order, that is when the coupling constant $\xi^{2}$ approaches its critical value $[24,44]$. Close to this point, the lightest $(l=0)$ mirror magnons condense, driving the system towards a new phase with gapless excitations. This is analogous to the transition from ferro- to antiferromagnetic order for compact spin chains in a magnetic field. It relates to the continuum limit of the fishnet graphs and to their correspondence with 2D $\sigma$ models with AdS target space. This correspondence, which was first discussed in Ref. [44] for $D=4$, also holds in higher dimensions. Namely, there is a dual set of TBA equations looking like that of the familiar $O(D+2) \sigma$ model in a finite volume $J$ except that instead of the standard relativistic dispersion relation we should use the one dual to Eq. (5).

The duality is established by means of the familiar particle-hole transformation. It involves the operator $\mathbb{1}-K_{O(D+2)}$ which solves the equation

$$
\left(\mathbb{1}-\mathcal{K}_{0,0}\right) \star\left(\mathbb{1}-K_{O(D+2)}\right)=\mathbb{1},
$$

with $\mathbb{1}$ the identity operator and with $\mathcal{K}_{0,0}$ as in Eq. (16). Straightforward algebra gives

$$
K_{O(D+2)}(u)=-i \partial_{u} \log S_{O(D+2)}(2 \pi u / D),
$$

with the well-known $O(D+2) S$ matrix [54]:

$S_{O(D+2)}(\theta)=-\frac{\Gamma\left(1+\frac{i \theta}{2 \pi}\right) \Gamma\left(\frac{1}{2}-\frac{i \theta}{2 \pi}\right) \Gamma\left(\frac{1}{D}-\frac{i \theta}{2 \pi}\right) \Gamma\left(\frac{1}{2}+\frac{1}{D}+\frac{i \theta}{2 \pi}\right)}{\Gamma\left(1-\frac{i \theta}{2 \pi}\right) \Gamma\left(\frac{1}{2}+\frac{i \theta}{2 \pi}\right) \Gamma\left(\frac{1}{D}+\frac{i \theta}{2 \pi}\right) \Gamma\left(\frac{1}{2}+\frac{1}{D}-\frac{i \theta}{2 \pi}\right)}$,

hinting at the dual $\sigma$-model interpretation.

Applying the operator $\mathbb{1}-K_{O(D+2)}$ to Eq. (13) for $l=0$ we get the dual equation for the scalar node:

$$
\log Y_{1,0}=J E-K_{O(D+2)} \star L_{1,0}^{\prime}-\sum_{l \geq 1} K_{1, l} \star L_{1, l},
$$

with $L_{1,0}^{\prime}=\log \left(1+Y_{1,0}^{-1}\right)$, and the new driving term

$$
E(u)=\log \left(\frac{\cosh \left(\frac{2 \pi u}{D}\right)+\cos \left(\frac{\pi \delta}{D}\right)}{\cosh \left(\frac{2 \pi u}{D}\right)-\cos \left(\frac{\pi \delta}{D}\right)}\right)
$$

is identified as the dual energy. As for the higher harmonics, Eqs. (13) for $l>0$, they can be rewritten as

$$
\log Y_{1, l}=-K_{l, 1} \star L_{1,0}^{\prime}-\sum_{l^{\prime} \geq 1} \check{K}_{l, l^{\star}} \star L_{1, l^{\prime}}+\sum_{l^{\prime} \geq 1} K_{l, l^{\prime}} \star L_{2, l^{\prime}} .
$$

The absence of driving terms in these equations indicates that the full symmetry is linearly realized in the dual picture. In fact, if not for the energy, Eqs. (22) and (24), as well as the ones in Eq. (15) which stay untouched, are identical to those for the $O(D+2) \sigma$ model.

The noncompactness of the model is seen in the fact that the spectrum is gapless, $\lim _{u \rightarrow \infty} E(u)=0$. This is made clearer after introducing a dual momentum $P$ obtained via a Wick rotation and a reflection $\delta \rightarrow \tilde{\delta}$ [61] 


$$
P(u)=-\left.i E(u+i D / 4)\right|_{\delta \rightarrow \tilde{\delta}} .
$$

It yields the dispersion relation

$$
\sinh ^{2} \frac{E}{2}=\tan ^{2}\left(\frac{\pi \tilde{\delta}}{D}\right) \sin ^{2} \frac{P}{2},
$$

as for a massless particle on a square lattice. It becomes relativistic at low energy, $E \sim c P$, that is when the continuous $\sigma$-model description applies, with the anisotropy being absorbed in the speed of light $c$.

One notices that the fishnet coupling constant $\xi^{2}$ disappears in the dual TBA equations. It is in line with the fact that the AdS $\sigma$ model admits no exactly marginal coupling. Instead, one finds by manipulating the original TBA Eqs. (13) that the coupling constant relates to the energy of the state $E_{2 \mathrm{D}}(\Delta, J)$ in the dual picture,

$$
E_{2 \mathrm{D}}(\Delta, J) \equiv-\int L_{1,0}^{\prime} \partial_{u} P(u) \frac{d u}{2 \pi}=J \log \frac{\xi^{2}}{\xi_{c}^{2}},
$$

with

$\log \xi_{c}^{2}=\int_{0}^{\infty}\left[\frac{D}{2} e^{-t}+\frac{e^{-\delta t}-e^{\delta t}+e^{-\tilde{\delta} t}-e^{\tilde{\delta} t}}{\left(1-e^{-t}\right)\left(1+e^{\frac{D t}{2}}\right)}\right] \frac{d t}{t}$,

the critical coupling. The latter controls the vacuum energy of the $\sigma$ model and thus the low-energy description corresponds to $\xi \sim \xi_{c}$, as alluded to before. Our expression for $\log \xi_{c}^{2}$ can be compared with Zamolodchikov's formula [24] for the free-energy density of a large fishnet graph. The agreement is perfect, for any $\delta$ and $D$, taking into account the normalization of the coupling constant.

Last, let us note that the TBA Eqs. (22), (24), and (15) can be brought, by inverting the kernels, to the $Y$-system form:

$$
\frac{Y_{a, l}^{[+]} Y_{a, l}^{[-]}}{Y_{a, l+1} Y_{a, l-1}}=\frac{\prod_{b=1}^{r}\left(1+Y_{b, l}\right)^{I_{a b}}}{\left(1+Y_{a, l+1}\right)\left(1+Y_{a, l-1}\right)},
$$

for all nodes with $1 \leq a \leq r, l \geq 1$ (with the convention that $Y_{a, 0}=\infty$ for $a>1$ ) while $Y_{1,0}$ satisfies

$$
\begin{aligned}
\frac{1}{Y_{1,0}^{[r-1]} Y_{1,0}^{[1-r]}}= & \prod_{k=1}^{r-2}\left(1+1 / Y_{r-k-1,1}^{[k]}\right)\left(1+1 / Y_{r-k-1,1}^{[-k]}\right) \\
& \times\left(1+1 / Y_{r-1,1}\right)\left(1+1 / Y_{r, 1}\right),
\end{aligned}
$$

with the shorthand notation $f^{[ \pm k]}(u)=f(u \pm i k / 2)$. This agrees with the $Y$-system equations of the $O(2 r)$ sigma model [62].

Excited states and asymptotic Bethe ansatz.-The TBA equations can be generalized to the states with an arbitrary number of magnons by the usual trick of the contour deformation [63-65]. The multimagnon operators $\mathcal{O}_{J, M}$, associated to spiral graphs shown in the right panel of Fig. 1, are made out of scalar magnons $(l=0)$ and obtained by exciting the corresponding $Y$ function. The $Y$-system relations stay the same as for the ground state, as well as most of the TBA formulas given earlier, if not for the energy (12) and Eqs. (13) which receive additional driving terms. The anomalous dimensions $\gamma_{M}=\Delta-(J \tilde{\delta}+M \delta)$ of the multimagnon states read then

$$
\gamma_{M}=\sum_{m=1}^{M}\left(2 i u_{m}-\delta\right)-\sum_{l \geq 0} \int p_{l}^{\prime}(u) L_{1, l}(u) \frac{d u}{2 \pi},
$$

with the $Y$ functions solving (13) with $\sum_{m} \log \mathbb{S}_{l, 0}\left(u, u_{m}\right)$ added in the right-hand side, and with the sums coming in both cases from the logarithmic poles at $Y_{1,0}\left(u_{m}\right)=-1$. The latter conditions are the exact Bethe ansatz equations, which reduce at large $J$ and for sufficiently weak coupling to the $\mathrm{ABA}$ equations

$$
1=\xi^{2 J} e^{-\varepsilon_{0}\left(u_{j}\right) J} \prod_{\substack{k=1 \\ k \neq j}}^{M} \mathbb{S}_{0,0}\left(u_{j}, u_{k}\right) .
$$

They are supplemented with the trace cyclicity condition

$$
\prod_{j=1}^{M} \xi^{2} e^{-\varepsilon_{0}\left(u_{j}\right)}=1 .
$$

This generalizes the 4D ABA equations of Ref. [6] to any dimension $D$ and any anisotropy.

As an example, in the simplest $M=1$ case, the ABA equation predicts that the anomalous dimension is given by $(2 i u-\delta)$, where $u$ is the solution to $\xi^{2} e^{-\varepsilon_{0}(u)}=1$. Expanding $u$ perturbatively in $\xi^{2}$ around the classical value $-i \delta / 2$, we find the one-magnon anomalous dimension

$\gamma_{M=1}=\frac{-2 \xi^{2}}{\Gamma\left(\frac{D}{2}\right)}+2 \xi^{4} \frac{\psi(\delta)+\psi(\tilde{\delta})-\psi\left(\frac{D}{2}\right)-\psi(1)}{\Gamma\left(\frac{D}{2}\right)^{2}}+O\left(\xi^{6}\right)$,

which agrees with the direct field theory computation.

As further checks, we considered two-magnon states for $J=5$. The corresponding mixing matrix takes the same form as for $D=4$ [6]. We have checked that the ABA prediction agrees with the direct diagrammatic computation through 3 loops.

Discussion and prospects.-We presented TBA equations for the exact spectrum of arbitrary multimagnon operators in the fishnet CFT in any spacetime dimension $D$. These operators form an important class of local operators of the theory and contain all of the information about the mirror dynamics. There are other types of operators worth being studied, including spinning operators (i.e., with 
derivatives) and the conjugate scalars $\bar{Z}, \bar{X}$. While it should be possible to include the former within the excited state TBA formalism, the latter are more elusive, and relate to the logarithmic property of the fishnet CFTs $[15,18,66]$.

The most efficient form of the TBA equations is expected to be given by Baxter equations. It would be good to derive them for generic $D$ and for a general local operator. This program is already quite advanced in $D=4$ case $[14,15,67]$, but not for other $D$ 's. Our TBA equations should help filling this gap by providing important information about the analyticity conditions and so-called quantization conditions specifying the solutions. The Baxter equations formulation would also be instrumental for a thorough study of the correspondence between fishnet graphs and noncompact sigma models or to reveal relationships with string-bit models in AdS [12-14]. Another interesting direction concerns the generalization of our TBAs to $\mathrm{FCFT}_{D}$ supported on triangular and hexagonal fishnets, or dynamical fishnet like the one found in the context of the three-coupling strongly twisted version of $\mathcal{N}=4$ SYM theory $[1,17]$.

A natural next step in the study of $\mathrm{FCFT}_{D}$ would be the computations of structure constants and multipoint correlation functions. In the mirror picture, it entails establishing the eigenfunctions of the graph-building operators in terms of Sklyanin separated variables, known so far only in two dimensions [41,68]; see also Ref. [69] for new developments. The formalism is closely related to the hexagon approach $[10,32,33,70]$ and would permit us to put it on a firmer ground for generic $D$. We believe that our two-body eigenfunction (6) is an important building block for constructing Sklyanin separated variables for noncompact quantum spin chains with arbitrary number of spins in principal series representations of $S O(1, D+1)$ symmetry [71], since these spin chains have the same integrability structure as $\mathrm{FCFT}_{D}$.

We thank Fedor Levkovich-Maslyuk for collaboration at an early stage of this project. We are thankful to János Balog, Johannes Henn, Shota Komatsu, Yang Zhang, and especially Dmitry Chicherin, Sergey Derkachov, and Enrico Olivucci for valuable comments and suggestions. We also thank Nikolay Gromov, Ivan Kostov, and Konstantin Zarembo for comments on the manuscript. The work of B. B. and D. 1.Z. was supported by the French National Agency for Research Grant No. ANR17-CE31-0001-02. The work of D. 1.Z. was supported in part by the center of excellence supported by the Israel Science Foundation (Grant No. 2289/18). D. 1. Z. is grateful to the Max Planck Institut für Physik and CERN for the warm hospitality during the final stage of this project.

Note added.-Recently, we learned [72] that Derkachev and Olivucci had obtained the two-body wave function (6) and its $M$-body generalization in a somewhat different form in the case of $D=4$. *benjamin.basso@ens.fr

†'gwenael.ferrando@ens.fr

*vladimir.kazakov@ens.fr

${ }^{\S}$ zdlzdlzdl@gmail.com

[1] Ö. Gürdoğan and V. Kazakov, Phys. Rev. Lett. 117, 201602 (2016); 117, 259903(A) (2016).

[2] V. Kazakov, Rev. Math. Phys. 30, 1840010 (2018).

[3] N. Gromov, V. Kazakov, and G. Korchemsky, J. High Energy Phys. 08 (2019) 123.

[4] C. Sieg and M. Wilhelm, Phys. Lett. B 756, 118 (2016).

[5] G. K. Karananas, V. Kazakov, and M. Shaposhnikov, arXiv:1908.04302.

[6] J. Caetano, Ö. Gürdoğan, and V. Kazakov, J. High Energy Phys. 03 (2018) 077.

[7] O. Mamroud and G. Torrents, J. High Energy Phys. 06 (2017) 012.

[8] D. Chicherin, V. Kazakov, F. Loebbert, D. Müller, and D.-1. Zhong, J. High Energy Phys. 05 (2018) 003.

[9] D. Chicherin, V. Kazakov, F. Loebbert, D. Müller, and D.-l. Zhong, Phys. Rev. D 96, 121901(R) (2017).

[10] B. Basso and L. J. Dixon, Phys. Rev. Lett. 119, 071601 (2017).

[11] D. Grabner, N. Gromov, V. Kazakov, and G. Korchemsky, Phys. Rev. Lett. 120, 111601 (2018).

[12] N. Gromov and A. Sever, Phys. Rev. Lett. 123, 081602 (2019).

[13] N. Gromov and A. Sever, J. High Energy Phys. 10 (2019) 085.

[14] N. Gromov and A. Sever, J. High Energy Phys. 02 (2020) 035.

[15] N. Gromov, V. Kazakov, G. Korchemsky, S. Negro, and G. Sizov, J. High Energy Phys. 01 (2018) 095.

[16] G. P. Korchemsky, J. High Energy Phys. 08 (2019) 028.

[17] V. Kazakov, E. Olivucci, and M. Preti, J. High Energy Phys. 06 (2019) 078.

[18] A. C. Ipsen, M. Staudacher, and L. Zippelius, J. High Energy Phys. 04 (2019) 044.

[19] R. de Mello Koch, W. LiMing, H. J. R. Van Zyl, and J. P. Rodrigues, Phys. Lett. B 793, 169 (2019).

[20] A. Pittelli and M. Preti, Phys. Lett. B 798, 134971 (2019).

[21] S. Dutta Chowdhury, P. Haldar, and K. Sen, J. High Energy Phys. 10 (2019) 249.

[22] T. Adamo and S. Jaitly, J. Phys. A 53, 055401 (2020).

[23] V. Kazakov and E. Olivucci, Phys. Rev. Lett. 121, 131601 (2018).

[24] A. B. Zamolodchikov, Phys. Lett. 97B, 63 (1980).

[25] The structure of such a graph near its boundary depends on the computed physical quantity.

[26] D. Chicherin, S. Derkachov, and A. P. Isaev, J. High Energy Phys. 04 (2013) 020.

[27] N. Beisert et al., Lett. Math. Phys. 99, 3 (2012).

[28] N. Beisert and M. Staudacher, Nucl. Phys. B727, 1 (2005).

[29] N. Beisert, B. Eden, and M. Staudacher, J. Stat. Mech. (2007) P01021.

[30] N. Gromov, V. Kazakov, and P. Vieira, Phys. Rev. Lett. 103, 131601 (2009).

[31] B. Basso, S. Komatsu, and P. Vieira, arXiv:1505.06745.

[32] T. Fleury and S. Komatsu, J. High Energy Phys. 01 (2017) 130.

[33] B. Eden and A. Sfondrini, J. High Energy Phys. 10 (2017) 098.

[34] D. Bombardelli, D. Fioravanti, and R. Tateo, J. Phys. A 42, 375401 (2009). 
[35] N. Gromov, V. Kazakov, A. Kozak, and P. Vieira, Lett. Math. Phys. 91, 265 (2010).

[36] G. Arutyunov and S. Frolov, J. High Energy Phys. 05 (2009) 068.

[37] N. Gromov, V. Kazakov, S. Leurent, and D. Volin, Phys. Rev. Lett. 112, 011602 (2014).

[38] N. Gromov, V. Kazakov, S. Leurent, and D. Volin, J. High Energy Phys. 09 (2015) 187.

[39] E. Panzer, Feynman integrals and hyperlogarithms, Ph.D. thesis, Humboldt University, Berlin, Inst. Math., 2015.

[40] D. J. Broadhurst, Phys. Lett. 164B, 356 (1985).

[41] S. Derkachov, V. Kazakov, and E. Olivucci, J. High Energy Phys. 04 (2019) 032.

[42] C. Ahn, Z. Bajnok, D. Bombardelli, and R. I. Nepomechie, J. High Energy Phys. 12 (2011) 059.

[43] For $D=3$, the ABJM model, in a similar double scaling limit, becomes a FCFT dominated by regular triangular planar graphs [6].

[44] B. Basso and D.-1. Zhong, J. High Energy Phys. 01 (2019) 002.

[45] N. Beisert, Adv. Theor. Math. Phys. 12, 948 (2008).

[46] R. A. Janik, Phys. Rev. D 73, 086006 (2006).

[47] N. Beisert, R. Hernandez, and E. Lopez, J. High Energy Phys. 11 (2006) 070.

[48] $\mathcal{H}_{D-\delta}$ is a Hilbert space with invariant inner product defined by $(f, g)=\int f^{*}\left(x_{1}\right) g\left(x_{2}\right)\left(x_{1}-x_{2}\right)^{-2 \delta} d^{D} x_{1} d^{D} x_{2}$.

[49] V. K. Dobrev, G. Mack, V. B. Petkova, S. G. Petrova, and I. T. Todorov, Lect. Notes Phys. 63, 1 (1977).

[50] When both spins are nonzero the integral over $x_{a}$ does not seem to be convergent. However, owing to the fact that standard manipulations allow us to formally prove that the full function is an eigenvector, we believe it should be understood as an analytic continuation in the exponents of the propagators. Similar manipulations, moreover, permit us to rewrite the function as a unique well-defined integral when one of the spins is zero.
[51] E. Ogievetsky and P. Wiegmann, Phys. Lett. 168B, 360 (1986).

[52] N. Y. Reshetikhin, Lett. Math. Phys. 7, 205 (1983).

[53] N. Y. Reshetikhin, Theor. Math. Phys. 63, 555 (1985).

[54] A. B. Zamolodchikov and A.B. Zamolodchikov, Ann. Phys. (N.Y.) 120, 253 (1979).

[55] M. Beneke and V. A. Smirnov, Nucl. Phys. B522, 321 (1998).

[56] S. E. Derkachov and A. N. Manashov, J. Phys. A 47, 305204 (2014).

[57] C.-N. Yang and C. P. Yang, J. Math. Phys. (N.Y.) 10, 1115 (1969).

[58] A. B. Zamolodchikov, Nucl. Phys. B342, 695 (1990).

[59] T. R. Klassen and E. Melzer, Nucl. Phys. B338, 485 (1990).

[60] T. R. Klassen and E. Melzer, Nucl. Phys. B350, 635 (1991).

[61] The reflection is needed because the square lattice is not invariant under a $\pi / 2$ rotation when there is anisotropy.

[62] J. Balog and Á. Hegedús, Nucl. Phys. B725, 531 (2005).

[63] P. Dorey and R. Tateo, Nucl. Phys. B482, 639 (1996).

[64] V. V. Bazhanov, S. L. Lukyanov, and A. B. Zamolodchikov, Nucl. Phys. B489, 487 (1997).

[65] D. Fioravanti, A. Mariottini, E. Quattrini, and F. Ravanini, Phys. Lett. B 390, 243 (1997).

[66] J. Caetano (private communication).

[67] D. Grabner, N. Gromov, V. Kazakov, and G. Korchemsky (to be published).

[68] S. E. Derkachov, G. P. Korchemsky, and A. N. Manashov, Nucl. Phys. B617, 375 (2001).

[69] N. Gromov, F. Levkovich-Maslyuk, P. Ryan, and D. Volin, Phys. Lett. B 806, 135494 (2020).

[70] B. Basso, J. Caetano, and T. Fleury, J. High Energy Phys. 11 (2019) 172.

[71] G. Ferrando (to be published).

[72] S. Derkachev and E. Olivucci (private communication). 